\title{
Laparoscopy and laparotomy in endometrial cancer - a meta-analysis
}

\author{
Emilia Tupacz-Mosakowska, Anna Abacjew-Chmyłko, Dariusz Wydra
}

Department of Gynaecology, Oncologic Gynaecology and Gynaecological Endocrinology, Medical University of Gdańsk, Poland

Submitted: 2 June 2019

Accepted: 22 May 2020

Arch Med Sci

DOI: https://doi.org/10.5114/aoms/122735

Copyright @ 2021 Termedia \& Banach

\begin{abstract}
Introduction: Uterine malignancies, the vast majority of which are endometrial cancers, constitute the most common type of gynecological neoplasms in developed countries. The primary treatment for endometrial cancer is hysterectomy and bilateral salpingoophorectomy. Women with endometrial cancer can be subjected to either total abdominal hysterectomy (TAH) or to an increasingly recommended total laparoscopic hysterectomy (TLH). We decided to verify whether published evidence supports TLH as an effective, less invasive than TAH albeit still equally radical treatment for endometrial malignancies.

Material and methods: The systematic review included articles indexed in MEDLINE (PubMed) and EBSCO, published between January 1974 and January 2017. The search was based on the following keywords and combinations thereof: "laparoscopy", "laparotomy", "endometrial cancer", "comparative". Twenty-six full-text articles were included in the meta-analysis.

Results: A total of 5,996 patients were eligible for the analysis, among them 2,833 (47.2\%) women subjected to TLH and 3,163 (52.8\%) who underwent TAH. Total laparoscopic hysterectomy is associated with shorter hospital stay, faster recovery, lesser blood loss and fewer intra- and postoperative blood transfusions, reduced pain, and a lower reoperation rate than conventional TAH.

Conclusions: All analyzed studies demonstrated that TLH is a safe and effective treatment option in endometrial cancer patients. This procedure is markedly less invasive than TAH. However, considering several contraindications for laparoscopy, such as peritoneal invasion, cardiorespiratory failure, history of previous surgery and large size of the uterus, qualification for a given procedure needs to be preceded by a detailed evaluation.
\end{abstract}

Key words: laparoscopy, endometrial cancer, laparotomy.

\section{Introduction}

Uterine malignancies, the vast majority of which are endometrial cancers (85\%), constitute the most common type of gynecological neoplasms in developed countries. Annually 189,000 new cases of endometrial cancer are registered worldwide, and yearly mortality due to this malignancy is estimated at 45,000 [1, 2]. The incidence rate of endometrial cancer in Poland is 14.8 per 100,000 and is higher than in Western European countries (nearly 12 per 100,000). The number of newly diagnosed endometrial cancers increased considerably in the period 1980-2000, and this tendency is expected to continue in the near future $[3,4]$.

\author{
Corresponding author: \\ Emilia Tupacz-Mosakowska \\ Department of Gynaecology, \\ Oncologic Gynaecology and \\ Gynaecological Endocrinology \\ Medical University of Gdańsk \\ 17 Smoluchowskiego St \\ 80-214 Gdańsk, Poland \\ E-mail: Emilia.tupacz@wp.pl
}


The primary treatment for endometrial cancer is hysterectomy and bilateral salpingoophorectomy, accompanied, in patients with an unfavorable profile of prognostic factors, by pelvic and paraaortic lymphadenectomy. Women with endometrial cancer can be subjected to either total abdominal hysterectomy (TAH) or, as is increasing ly recommended, to total laparoscopic hysterectomy (TLH).

Endometrial cancer is most commonly diagnosed in high-risk patients - women with overweight/obesity and multiple comorbidities, such as arterial hypertension and diabetes mellitus.

The choice of an adequate treatment method is a key determinant of 5-year survival in cancer patients. The decision whether to choose a given therapeutic option is based on three groups of factors: patient-dependent (such as general condition and comorbidities), tumor-dependent (stage, histological type) and medical center-dependent $[3,5]$. Another factor that needs to be considered is the improvement of patients' quality of life during the peri- and postoperative period; this can be achieved by shorter hospital stay, decreased morbidity, faster healing of the surgical wound, better control of pain and lower demand for postoperative analgesia, reduced blood loss

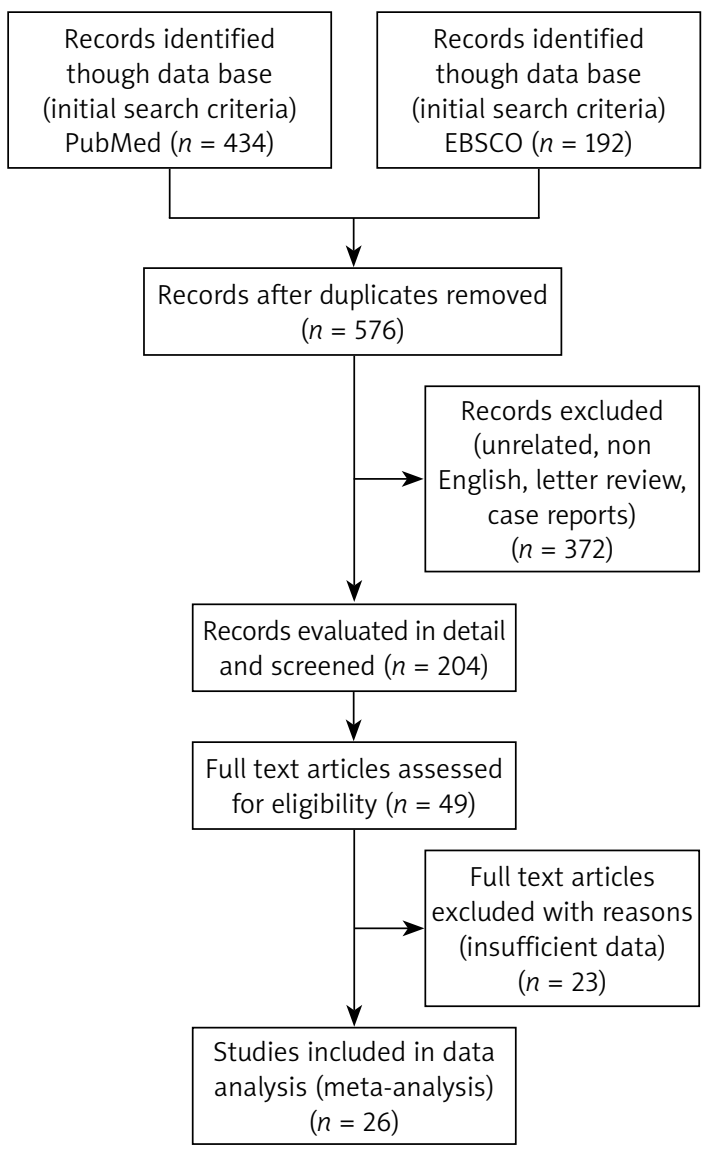

Figure 1. Flowchart for selecting relevant articles and fewer blood transfusions, prompt recovery and earlier return to normal physical activity. All these criteria can be satisfied by laparoscopic techniques, which are gaining growing popularity as an endometrial cancer treatment. However, a prerequisite for the use of those minimally invasive techniques is adequate quality of staging and radicality of the resection, both resembling those achieved during conventional laparotomy. Therefore, we decided to verify whether published evidence supports TLH as an effective, less invasive than $\mathrm{TAH}$ albeit still equally radical treatment for endometrial malignancies.

The aim of this systematic review and metaanalysis was to compare the outcomes of TAH and TLH in endometrial cancer patients.

\section{Material and methods}

The systematic review included articles indexed in MEDLINE (PubMed) and EBSCO, published between January 1974 and January 2017. The search was based on the following keywords and combinations thereof: 'laparoscopy', 'laparotomy', 'endometrial cancer', 'comparative'. The results were limited to articles published in English. Reference lists from all identified publications and available review articles were also searched manually.

Of 572 records initially identified, a total of 204 articles were identified after assessment of their titles and/or abstracts for eligibility. All these papers satisfied the following criteria: (1) included endometrial cancer patients, (2) compared women subjected to laparoscopic (TLH) and laparotomic (TAH) resection of the uterus and regional lymph nodes, and (3) analyzed early outcomes of the surgical treatment, such as operative time, blood loss, length of hospital stay, intra- and postoperative morbidity, relaparotomy rates and conversions to laparotomy during laparoscopic procedures. Eventually, 26 full-text articles were available for meta-analysis. Studies analyzing the outcomes of transcervical endometrial resection under laparoscopic guidance were not included in the analysis. However, the analysis included studies of patients with atypical endometrial hyperplasia as this condition is a precursor lesion for endometrial cancer and may co-exist with this malignancy in $15 \%$ of cases; moreover, patients with either atypical endometrial hyperplasia or endometrial cancer present with similar clinical phenotype, i.e. similar comorbidities and comparable surgical risk. Moreover, the analysis included eight studies comparing conventional laparoscopy (TLH) and laparotomy (TAH) with total robotic hysterectomy (TRH).

The meta-analysis was conducted in accordance with the Meta-analysis of Observational Studies in Epidemiology (MOOSE) statement [6]. 
Table I. Comparison of the number of patients and the age for particular types of surgery - laparoscopy and laparotomy $(p<0.05$ in bold $)$

\begin{tabular}{|c|c|c|c|c|c|c|c|c|}
\hline \multirow{3}{*}{$\begin{array}{l}\text { Authors } \\
\text { of the publication }\end{array}$} & \multicolumn{3}{|c|}{ Number of patients } & \multicolumn{5}{|c|}{ Age [years] } \\
\hline & \multirow[t]{2}{*}{ TLH, $n$} & \multirow[t]{2}{*}{ TAH, $n$} & \multirow[t]{2}{*}{$\Delta(\%)$} & \multicolumn{2}{|c|}{ TLH } & \multicolumn{2}{|c|}{ TAH } & \multirow[t]{2}{*}{$P$-value } \\
\hline & & & & Mean & SD (range) & Mean & SD (range) & \\
\hline Barnett et al. [29]\& & 107 & 269 & 60.2 & - & - & - & - & 0.94 \\
\hline $\begin{array}{l}\text { Barwijuk and } \\
\text { Jankowska [20] }\end{array}$ & 12 & 13 & 7.7 & 62 & $(40-91)$ & 62 & $(37-83)$ & - \\
\hline Bell et al. [25] & 30 & 40 & 25.0 & 68.4 & 11.9 & 72.3 & 12.5 & 0.03 \\
\hline Berretta et al. [24] & 27 & 27 & 0.0 & 67 & $(44-77)$ & 63 & $(49-77)$ & 0.45 \\
\hline Bige et al. [34] & 70 & 70 & 0.0 & 55.56 & 10.62 & 56.24 & 10.55 & NS \\
\hline Boggess et al. [19] & 81 & 138 & 41.3 & 62.0 & 10.8 & 64.0 & 12.8 & 0.06 \\
\hline Boosz et al. [26] & 107 & 160 & 33.1 & 63.2 & 11.0 & 66.7 & 11.3 & 0.01 \\
\hline Chiou et al. [28] & 150 & 129 & 14.0 & 51.4 & 14.2 & 53.6 & 11.3 & 0.73 \\
\hline Chu et al. [33] & 70 & 81 & 13.6 & 55.3 & $(29-80)$ & 53.4 & $(28-75)$ & 0.248 \\
\hline Coronado et al. [30] & 84 & 192 & 56.3 & 65.9 & 11.2 & 64.7 & 11.2 & 0.245 \\
\hline Corrado et al. [11] & 277 & 177 & 36.1 & 62 & $(28-86)$ & 64 & $(35-90)$ & 0.08 \\
\hline Eisenhauer et al. [18] & 25 & 154 & 83.8 & 57 & $(35-79)$ & 60 & $(25-84)$ & 0.11 \\
\hline Eisenkop [31] & 210 & 246 & 14.6 & 63.6 & 13.6 & 65.2 & 11.3 & 0.248 \\
\hline Gao and Zhang [21] & 81 & 81 & 0.0 & 57.02 & 1.06 & 57.64 & 1.16 & 0.69 \\
\hline Jung et al. [10] & 25 & 56 & 55.4 & 49.9 & 10.75 & 50.2 & 8.06 & 0.164 \\
\hline Lim et al. [23] & 56 & 36 & 35.7 & 61.4 & 11.7 & 62.7 & 10.6 & 0.77 \\
\hline Lu et al. [9] ${ }^{\mathrm{R}}$ & 151 & 121 & 19.9 & 56.6 & $(27-82)$ & 57.2 & $(29-79)$ & 0.11 \\
\hline Malzoni et al. $[14]^{R}$ & 81 & 78 & 3.7 & 60 & 11 & 63 & 14 & NS \\
\hline Manchana et al. [27] & 47 & 143 & 67.1 & 54 & $(49-62)$ & 59 & $(53-65)$ & $<0.01$ \\
\hline Mourits et al. [15] $]^{\mathrm{R}}$ & 185 & 94 & 49.2 & 62 & $(40-89)$ & 63 & $(39-86)$ & - \\
\hline Obermair et al. $[16]^{\& R}$ & 404 & 349 & 13.6 & - & - & - & - & - \\
\hline O’Hanlan et al. [17] & 76 & 29 & 61.8 & 60.9 & 13.1 & 67.6 & 13.0 & 0.021 \\
\hline Pellegrino et al. [13] & 37 & 37 & 0.0 & 54 & - & 54 & - & - \\
\hline Qviqstad and Lieng [12] & 281 & 230 & 18.1 & - & $(57-65)$ & - & $(62-68)$ & - \\
\hline Santi et al. [22] & 120 & 120 & 0.0 & 62 & - & 63 & - & - \\
\hline Terai et al. [8] & 39 & 93 & 58.1 & 56.6 & 10 & 56.2 & 11.6 & 0.86 \\
\hline
\end{tabular}

The literature review search showing the process of inclusion and exclusion based on PRISMA guidelines $[6,7]$ is presented in a flow-chart in Figure 1.

\section{Results}

Eventually, a total of 5,996 patients were eligible for the analysis, among them 2,833 (47.2\%) women subjected to TLH and 3,163 (52.8\%) who underwent TAH (Table I).

\section{Research reliability}

Three parameters of research reliability were analyzed: 1) size of the groups, 2) type of research, 3) consistency of the compared groups.

\section{Size of the groups}

In the 9 out of 26 studies both TLH and TAH groups included over 100 patients [8, 9, 11, 12, 16, $26,28,29,31]$, in 12 studies the analyzed groups 
Table II. Statistical differences between the characteristics of patients and surgical outcomes in the laparoscopy and laparotomy groups $(p<0.05$ in bold)

\begin{tabular}{|c|c|c|c|c|c|c|c|c|c|c|c|}
\hline \multirow[t]{2}{*}{$\begin{array}{l}\text { Authors of } \\
\text { the publication }\end{array}$} & \multirow[t]{2}{*}{ Age } & \multirow[t]{2}{*}{ BMI } & \multirow{2}{*}{$\begin{array}{l}\text { Histo- } \\
\text { logical } \\
\text { type }\end{array}$} & \multirow[t]{2}{*}{$\begin{array}{l}\text { FIGO } \\
\text { stage }\end{array}$} & \multirow[t]{2}{*}{$\begin{array}{l}\text { Nodal } \\
\text { status }\end{array}$} & \multirow[t]{2}{*}{ Grade } & \multirow{2}{*}{$\begin{array}{c}\text { Mean } \\
\text { pelvic } \\
\text { nodes } \\
\text { removed }\end{array}$} & \multirow{2}{*}{$\begin{array}{c}\text { Mean } \\
\text { aortic } \\
\text { nodes } \\
\text { removed }\end{array}$} & \multicolumn{3}{|c|}{$\begin{array}{c}\text { Frequency } \\
\text { of lymphadenectomy }\end{array}$} \\
\hline & & & & & & & & & TLH & TAH & $P$-value \\
\hline $\begin{array}{l}\text { Barnett et al. } \\
\text { [29] }\end{array}$ & 0.94 & 0.92 & 0.732 & - & - & 0.04 & 0.74 & 0.41 & 22 & 14.8 & - \\
\hline $\begin{array}{l}\text { Barwijuk and } \\
\text { Jankowska [20] }\end{array}$ & - & - & - & - & - & - & - & - & - & - & - \\
\hline Bell et al. [25] & 0.03 & NS & - & - & - & - & - & - & - & - & - \\
\hline $\begin{array}{l}\text { Berretta et al. } \\
{[24]}\end{array}$ & 0.45 & 0.38 & - & NS & - & NS & - & - & - & - & - \\
\hline Bige et al. [34] & NS & NS & NS & NS & NS & NS & - & NS & - & - & - \\
\hline $\begin{array}{l}\text { Boggess et al. } \\
{[19]}\end{array}$ & 0.06 & 0.17 & - & - & - & - & $<0.0001$ & $<0.0001$ & - & - & - \\
\hline $\begin{array}{l}\text { Boosz et al. } \\
{[26]}\end{array}$ & 0.01 & 0.09 & - & $<0.00001$ & $<0.0001$ & $<0.01$ & $<0.001$ & 0.13 & - & - & - \\
\hline $\begin{array}{l}\text { Chiou et al. } \\
{[28]}\end{array}$ & 0.73 & 0.85 & 0.32 & 0.12 & 0.69 & - & - & - & - & - & - \\
\hline Chu et al. [33] & 0.248 & 0.354 & - & 0.123 & 0.213 & 0.296 & - & - & - & - & - \\
\hline $\begin{array}{l}\text { Coronado et al. } \\
\text { [30] }\end{array}$ & 0.245 & 0.016 & 0.553 & 0.441 & - & 0.694 & 0.461 & 0.238 & 61.9 & 66.1 & 0.7 \\
\hline $\begin{array}{l}\text { Corrado et al. } \\
\text { [11] }\end{array}$ & 0.08 & 0.06 & 0.62 & 0.27 & 0.89 & 0.05 & - & - & 32.5 & 36.1 & 0.89 \\
\hline $\begin{array}{l}\text { Eisenhauer et } \\
\text { al. [18] }\end{array}$ & 0.11 & $<0.001$ & 0.88 & 0.28 & 0.002 & 0.42 & 0.001 & 0.18 & 40 & 45 & - \\
\hline Eisenkop [31] & 0.248 & 0.830 & 0.054 & 0.920 & $<0.001$ & 0.107 & $<0.001$ & $<0.001$ & - & - & - \\
\hline $\begin{array}{l}\text { Gao and Zhang } \\
{[21]^{*}}\end{array}$ & 0.69 & 0.91 & 0.84 & 0.69 & 0.44 & 0.47 & $<0.01$ & 0.11 & 100 & 100 & - \\
\hline Jung et al. [10] & 0.164 & 0.468 & 0.181 & 0.437 & - & - & 0.024 & 0.066 & - & - & - \\
\hline Lim et al. [23] & 0.77 & 0.45 & - & - & $<0.001$ & - & $<0.001$ & $<0.001$ & - & - & - \\
\hline Lu et al. [9] ${ }^{R}$ & 0.11 & 0.25 & 0.65 & 0.15 & 0.67 & 0.75 & 1.00 & 0.03 & - & - & - \\
\hline $\begin{array}{l}\text { Malzoni et al. } \\
{[14]^{R}}\end{array}$ & NS & NS & NS & NS & NS & NS & NS & NS & 0 & 0 & - \\
\hline $\begin{array}{l}\text { Manchana et } \\
\text { al. [27] }\end{array}$ & $<.01$ & - & - & - & - & - & - & - & 2 & 14.7 & 0.02 \\
\hline $\begin{array}{l}\text { Mourits et al. } \\
{[15]^{R}}\end{array}$ & - & - & - & - & $x$ & $x$ & $x$ & $x$ & - & - & - \\
\hline $\begin{array}{l}\text { Obermair et al. } \\
{[16]^{\mathrm{R}}}\end{array}$ & - & - & - & - & - & - & - & - & 39.9 & 60.2 & $<0.001$ \\
\hline O’Hanlan [17] & 0.021 & - & - & - & - & - & - & - & 28 & 79 & $<0.001$ \\
\hline Pellegrino [13] & - & NS & NS & - & - & - & - & - & 65 & 57 & - \\
\hline Qviqstad [12] & - & - & - & - & - & - & - & - & 0 & 0 & - \\
\hline Santi [22] & - & NS & - & NS & $<0.05$ & - & - & - & - & - & - \\
\hline Terai [8] & 0.86 & 0.025 & - & 0.71 & 0.15 & 0.10 & - & - & - & - & - \\
\hline
\end{tabular}

(at least one of the two) included between 40 and 100 patients $[8,10,14,15,17,19,21,23,27,30$, $33,34]$, and in another four studies both had no more than $40[13,20,24,25]$. There was a single large series presented by Obermair et al. [16] of 404 women subjected to TLH and 349 subject- 


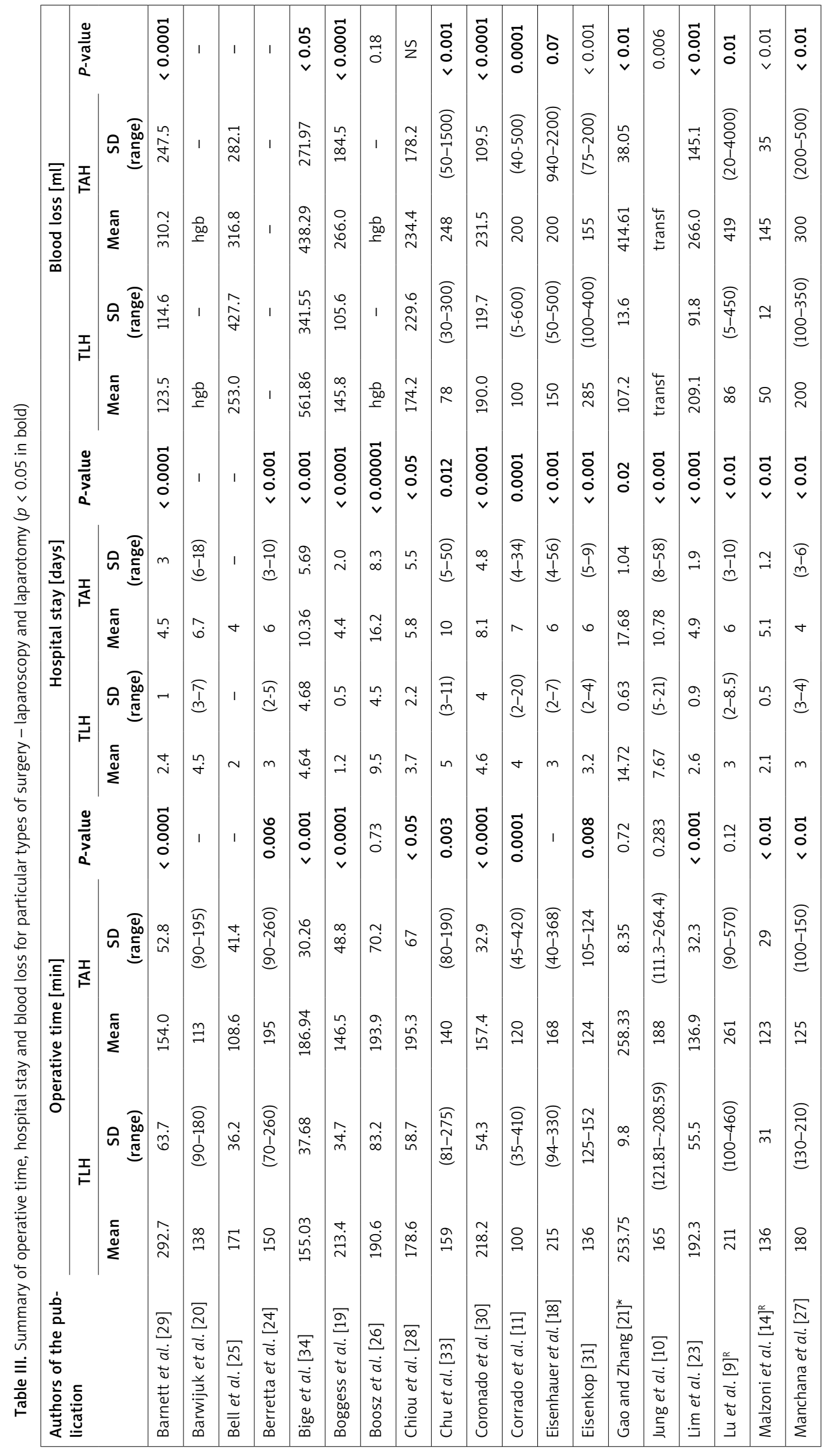




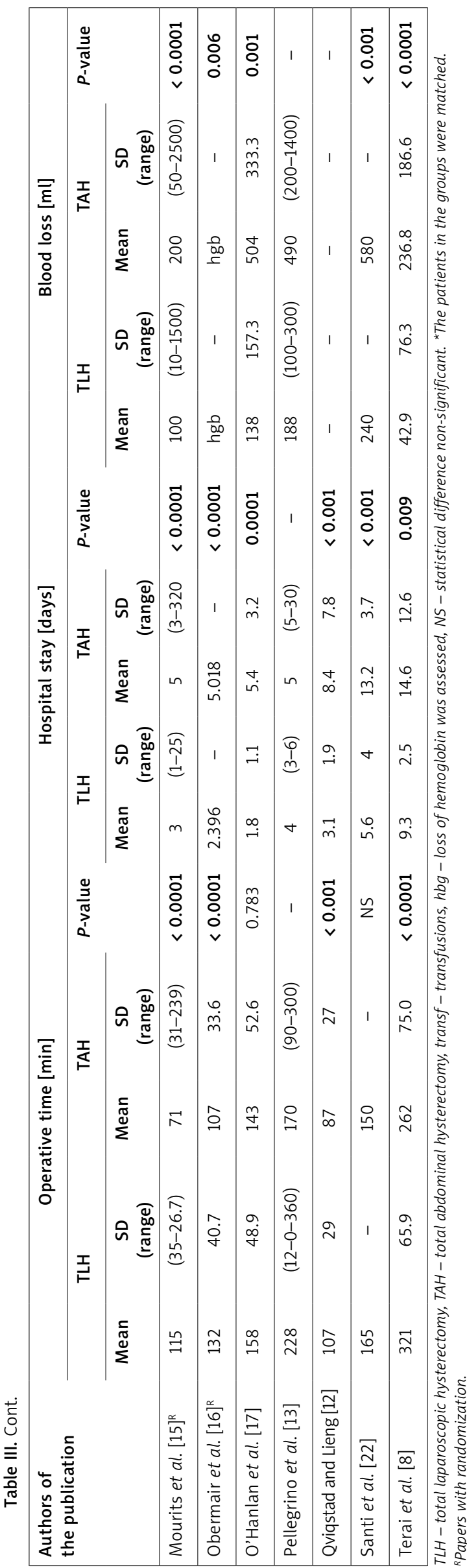

ed to TAH. Thirteen 13 studies had a similar number of patients (with the mathematical difference not exceeding 25\%) in the corresponding study groups; in the rest the differences reached up to 67\% (Table I).

\section{Type of research}

Most analyzed publications were based on single-center studies conducted at clinics claiming to have extensive experience with laparoscopic procedures; only five papers presented the results of multicenter studies [15-17, 30, 34]. In four studies, patients were randomized to either TLH or TAH $[9,14-16]$.

\section{Consistency of the compared groups}

Table II presents an assessment of how homogeneous the TLH and TAH groups have been in particular studies. A majority of the papers evaluated the uniformity of patient characteristics (age and $\mathrm{BMI})$, finding differences only in a few $[17,26,27]$. Only 17 papers performed an evaluation of the tumor-related characteristics (histological type, tumor grading, FIGO stage and metastases in lymph nodes), but not all were complete. Eight papers lacked any comparison [12, 15-17, 19, 20, 25, 27]. In the randomized studies [9, 14-16], although not mandatory, only two $[9,14]$ out of four assessed the statistical differences between groups in both patient and tumor characteristics, demonstrating no statistical differences. Another paper by Gao et al. [21] included TLH and TAH groups matching on the basis of their characteristics, which eliminated differences between groups. As they described, propensity scores were calculated using a nonparsimonious multivariable logistic regression model to estimate the conditional probability of a patient receiving a surgery approach. Then a 1 : 1 match between the laparoscopy and laparotomy groups was performed using nearest available neighbor matching.

In studies without randomization and without matching, the process of assignment to individual research groups was carried out on the basis of very different criteria. The authors of 5 studies, however, did not provide information about the procedure of qualification for surgery [24-28]. In the remaining studies, patients were excluded from the analysis whenever they presented with concomitant ovarian cancer and underwent a neoadjuvant therapy [14, 27, 29], and/or were diagnosed with stage III or IV endometrial cancer $[8,10,12-16,18,20,22,24,31,33,34]$. The statistical comparison of the FIGO stage distribution between laparoscopy and laparotomy groups has been assessed in 13 studies and only Boosz et al. [26] stated that more advanced endometrial 
Table IV. Long-term treatment results $(p<0.05$ in bold)

\begin{tabular}{|c|c|c|c|c|c|}
\hline $\begin{array}{l}\text { Authors of } \\
\text { the publication }\end{array}$ & $\begin{array}{c}\text { Follow-up period (median) } \\
\text { [months] }\end{array}$ & $\begin{array}{l}\text { Recurrence, } \\
n(\%)\end{array}$ & $\begin{array}{l}\text { Death, } \\
n\end{array}$ & $\begin{array}{c}\text { Disease-free survival } \\
\quad(5 \text { years })\end{array}$ & $\begin{array}{c}\text { Overall survival } \\
\text { (\%) }\end{array}$ \\
\hline \multicolumn{6}{|l|}{ Bige et al. [34] } \\
\hline TLH & 31.14 & 0 & 1 & $100.00 \%$ (3 years) & 97.14 \\
\hline TAH & 34.80 & $1(1.42)$ & 2 & $98.57 \%$ (3 years) & 98.57 \\
\hline$P$-value & NS & NS & NS & NS & NS \\
\hline \multicolumn{6}{|l|}{ Chiou et al. [28] } \\
\hline TLH & 18.00 & $7(4.60)$ & - & - & 98.00 \\
\hline TAH & 18.00 & $6(5.00)$ & - & - & 94.60 \\
\hline$P$-value & - & - & - & - & 0.09 \\
\hline \multicolumn{6}{|l|}{ Chu et al. [33] } \\
\hline TLH & 59.00 & $2(2.90)$ & $1(1.40)$ & $97.10 \%$ & 98.60 \\
\hline TAH & 59.00 & $4(4.90)$ & $1(1.20)$ & $96.30 \%$ & 97.50 \\
\hline$P$-value & - & 0.514 & 0.917 & - & - \\
\hline \multicolumn{6}{|c|}{ Coronado et al. [30] } \\
\hline TLH & 20.70 & 7 & 0 & $83.10 \%$ (3 years) & 100.00 \\
\hline TAH & 20.70 & 5 & 2 & $88.70 \%$ (3 years) & 93.60 \\
\hline$P$-value & - & - & - & 0.838 & 0.248 \\
\hline \multicolumn{6}{|c|}{ Corrado et al. [11] } \\
\hline TLH & 47.00 & $20(7.20)$ & 8 & $88.40 \%$ (3 years) & 91.70 \\
\hline TAH & 78.00 & $15(11.90)$ & 5 & 92.10\% (3 years) & 86.70 \\
\hline$P$-value & 0.001 & - & - & - & - \\
\hline \multicolumn{6}{|c|}{ Gao and Zhang [21] } \\
\hline TLH & 45.00 & $9(11.10)$ & 9 & $85.30 \%$ & 85.30 \\
\hline $\mathrm{TAH}$ & 45.00 & $7(8.60)$ & 11 & $89.20 \%$ & 75.80 \\
\hline$P$-value & - & 0.6 & - & - & 0.97 \\
\hline \multicolumn{6}{|l|}{ Lu et al. [9] } \\
\hline TLH & 68.00 & $7(4.60)$ & 9 & $96.00 \%$ & 94.00 \\
\hline $\mathrm{TAH}$ & 68.00 & $6(5.00)$ & 12 & $91.00 \%$ & 90.10 \\
\hline$P$-value & - & NS & - & - & 0.418 \\
\hline \multicolumn{6}{|c|}{ Malzoni et al. [14] } \\
\hline TLH & 38.50 & $7(8.60)$ & 5 & $91.40 \%$ & 93.20 \\
\hline $\mathrm{TAH}$ & 38.50 & $9(11.50)$ & 7 & $88.50 \%$ & 91.10 \\
\hline$P$-value & - & NS & - & 0.28 & 0.31 \\
\hline \multicolumn{6}{|c|}{ Manchana et al. [27] } \\
\hline TLH & 24.00 & $2(4.20)$ & 0 & - & - \\
\hline $\mathrm{TAH}$ & 20.00 & $15(10.50)$ & 6 & - & - \\
\hline$P$-value & - & 0.52 & - & - & - \\
\hline
\end{tabular}

TLH - total laparoscopic hysterectomy, TAH - total abdominal hysterectomy, NS - statistical difference non-significant. ${ }^{R}$ Papers with randomization 
malignancies were assigned to the TAH group (Table II). Similarly, two other studies included patients with more advanced endometrial malignancies [17, 29]; such women were more often qualified for TAH than for TLH. In the study conducted by O'Hanlan et al. [17], malignancies with FIGO stage II or higher were found in 35\% of women subjected to TAH and in $17 \%$ of patients who underwent TLH. In the study conducted by Barnett et al. [29], 52\% of women subjected to TLH and $39 \%$ of those who underwent $\mathrm{TAH}$ presented with FIGO stage I malignancies. However, in neither of these studies was a statistical evaluation of FIGO staging distribution performed (Table II).

Positive intraoperative peritoneal lavage cytology, still an important prognostic factor, was discussed only in the O'Hanlan study [17]. It was documented in a similar proportion of patients subjected to TAH and TLH (in $22 \%$ and $15 \%$, respectively).

Due to the non-homogeneous methodology of presenting patients' age, we analyzed only those studies in which this parameter was described as a median. In those studies, the median age of patients subjected to TLH and TAH was 61 years (range 27-91) and 63 years (range 25-90), respectively, with only 4 studies [17, 25-27] showing an age difference between groups (Table II).

In 13 studies (46.2\%), patients were homogeneous in terms of their histopathological diagnoses and presented solely with endometrial cancer $[8,10,13,16,19,20-26,33]$. In another 13 studies (54.2\%), the outcomes in endometrial cancer patients were compared with the results of women with uterine clear-cell, serous and mixed carcinomas [9, 11, 14, 21, 27-29, 34], endometrial hyperplasia [12] or uterine carcinosarcoma [18, 31]. The differences of histopathological distribution were not found to be significant between the TLH and TAH groups (Table II).

The list of factors that favored the qualification of endometrial cancer patients for TLH included:

- lower clinical stage [17, 26, 29],

- higher body weight [8, 18, 27, 30]; although in most studies, the proportions of overweight/ obese patients qualified for TLH and TAH were essentially the same [8-11, 13, 14, 18, 19, 23-26, 28-31, 33, 34]; in particular Malzoni et al. [14] disqualified all patients with $\mathrm{BMI}>40 \mathrm{~kg} / \mathrm{m}^{2}$ from the laparoscopic procedure.

In turn, TAH was a preferred treatment in patients with:

- older age [17, 26, 27], larger uterus [9, 14, 15, 17, 21], greater depth of myometrial invasion [17], metastases to regional lymph nodes $[17,18,23$, $26,31]$, high-grade endometrial malignancies $[26,29]$.
The following groups of patients were disqualified from TLH:

- with contraindications to anesthesia (COPD, cardiovascular diseases) [9, 14, 15, 21],

- with a history of past laparotomies and resultant increased risk of postsurgical intraperitoneal adhesion formation [17],

- with a large uterus (with estimated weight $>250 \mathrm{~g}$ or size greater than at 12 weeks of gestation) $[9,14,15,21]$,

- with life expectancy of less than 6 months [16], or older than 80 years [14].

Additionally, Obermair et al. [16] excluded from the analysis all women who did not refer for the scheduled postoperative follow-up visits.

Only the Chinese study [21] evaluated the safety of treatment in high-risk patients, i.e. with $\mathrm{G} 2$ or G3 malignancies, non-endometroid carcinomas, deep myometrial invasion (more than 50\% of myometrial thickness), metastases to pelvic and paraaortic lymph nodes, and FIGO stages II-IV. They concluded that TLH and TAH can be safely performed in high-risk patients.

A single study speculated about an unfavorable effect of hysteroscopy as a preoperative diagnostic method in patients with suspected endometrial cancer. O'Hanlan et al. [17] stated that hysteroscopy poses a risk of extruding cancer cells to the peritoneal cavity via the fallopian tubes. In the study conducted by those authors, $22 \%$ of women subjected by $\mathrm{TAH}$ and $15 \%$ of patients who underwent TLH had positive intraoperative peritoneal lavage cytology; however, due to the lack of exact data on preoperative hysteroscopy rates, peritoneal invasion in those patients could not be unequivocally qualified as an adverse effect of this diagnostic procedure. Preventive measures undertaken to minimize the risk of peritoneal seeding included placement of the uterine manipulator after coagulation of the fallopian tubes, and avoidance of excessive uterine manipulation during $\operatorname{TLH}[9,10,14,20]$.

\section{Lymph node dissection and lymph node status}

The criteria for lymph node dissection were defined only in 3 papers $[8,16,17]$. Lymphadenectomy was not performed in patients with G1 malignancies and in women in whom myometrial invasion did not exceed $50 \%$ of myometrial thickness. However, all patients with G3 neoplasms, as well as women with G1 or G2 malignancies and myometrial invasion greater than $50 \%$ of myometrial thickness, were qualified for iliac and paraaortic lymphadenectomy $[8,16,17]$. Only two studies mentioned that the degree of myometrial invasion was evaluated intraoperatively in all patients with G1 or G2 tumors [16, 17]. The Malzoni study [14] 
enrolled for randomization high risk early-stage patients; thus all had pelvic lymphadenectomy. In this study para-aortic lymphadenectomy was performed in cases with positive pelvic lymph nodes discovered at frozen section evaluation, in patients with poorly differentiated tumors with myometrial invasion greater than 50\% (ICG3), and non-endometrioid carcinomas. Maurits et al. [15] and Qviqsrad et al. [12] did not enroll lymphadenectomy patients in the randomization study at all. In two other studies postoperative qualification for adjuvant treatment was described - patients with low-grade uterine malignancies without regional lymph node involvement did not receive adjuvant treatment $[8,15]$.

The extent of pelvic and paraaortic lymphadenectomy (considered as the mean number of lymph nodes removed) was analyzed in 11 studies (Table II). In 4 studies, the overall number of removed nodes was similar regardless of the surgical method. In the study conducted by Jung et al. [10], the overall number of nodes removed during TLH was significantly lower than in the case of TAH $(18.36 \pm 7.25$ vs. $24.39 \pm 10.08)$; the two groups did not differ in the number of removed paraaortic nodes. Similar results were also reported by Boosz et al. [26] and Eisenhauer et al. [18]. In the studies conducted by Lim et al. [23], Boggess et al. [19] and Eisenkop [31] the mean number of pelvic nodes and paraaortic nodes removed in women subjected to TAH was significantly higher than in those who underwent TLH. Neither of the two randomized studies $[9,14]$ showed any difference in the number of harvest lymph nodes in both surgical groups; however, the other two $[15,16]$ did not analyze the difference. Addressing this problem, Barnett et al. [29] also analyzed the learning curve for laparoscopic lymphadenectomy and found that the number of lymph nodes removed during a single TLH procedure increased over time, from 5 in 2002 to 15-20 in 2007 [29]. Despite a greater number of harvested lymph nodes in laparoscopy, Gao et al. found that the difference in pelvic lymph node count between the laparoscopy and laparotomy groups did not influence the overall survival [21].

A larger number of harvested lymph nodes was found to correlate with the nodal status [18, $23,26,31]$. In the series examined by Boosz et al. [26], metastases to regional lymph nodes were found more frequently in patients subjected to $\mathrm{TAH}$ ( $n=26,16.2 \%$ vs. $n=4,3.7 \%$ in TLH group). However, the authors attributed that difference to a higher clinical stage of endometrial cancers in patients subjected to TAH.

The rates of lymphadenectomy performed in the studies differed between laparoscopy and laparotomy groups, and also between papers (Table II).
In three studies $[16,17,27]$ regional lymphadenectomy was performed with a significantly lower rate during laparoscopy than during laparotomy. In the Obermair et al. study [16] there were no data collected on the reason for surgeons' decision for or against a lymph node dissection, but they mentioned that the decision not to proceed with a node dissection in laparoscopic cases was most likely based on the feasibility of a node dissection in obese and super-obese patients. Pellegrini et al. [13] compared the outcomes of laparoscopic and laparotomic lymphadenectomy in obese and normal-weight patients, yet found that the effectiveness of lymphadenectomy was independent of patient's BMI.

Some authors outlined the benefits of laparoscopic lymphadenectomy. According to Gao et al. [21], laparoscopic lymphadenectomy can be more effective due to better visualization of the operated site. Barnett et al. [29] reported that removal of the most distally located iliac nodes and their rich lymphatic network may result in lower limb lymphedema [29]. Moreover, they postulated that pelvic lymphadenectomy may pose a risk of genitofemoral nerve injury and resultant sensory deficits, and therefore better visualization of the surgical field during TLH may contribute to more "aggressive" dissection of tissues.

\section{Operative time}

In most studies (18/26), including three studies performed with randomization [14-16], the mean duration of TLH was longer than the mean operative time of TAH (Table III). In six studies [9-11, $17,22,26]$ the surgical methods did not differ significantly in terms of the operative time. In three studies [11, 24, 28] TLH lasted shorter than TAH.

Distribution of surgical time has been very wide, in the range 35-460 min for TLH and 40-570 min for $\mathrm{TAH}$. It needs to be stressed that the methodology used to determine the operative time varied from study to study. While some authors counted duration of the procedure from the first incision of the skin, others used the onset of anesthesia as the reference timepoint. Preparation of instruments for TLH was shown to contribute to longer duration of the procedure [14]. Additionally, in many papers the operative time was compared regardless of the extent of surgical procedures. Only Coronado et al. [30] estimated operative times for the procedures with and without regional lymphadenectomy at $239.6 \mathrm{~min}$ and $169.2 \mathrm{~min}$, respectively, for TLH, and at $189.5 \mathrm{~min}$ and 135.4 min, respectively, for TAH $(p<0.0001)$.

Another factor influencing the large distribution of the operative time is the experience of the surgical team, as the time decreased with the number of performed procedures, which has 
been independently demonstrated by Gao and Zhang [21] and O'Hanlan et al. [17].

\section{Blood loss}

All studies but two $[18,26]$ demonstrated that TLH was associated with lesser blood loss than TAH (Table III). However, the methodology used to estimate the degree of blood loss varied, from subjective estimation (volume count) done by an anesthesiologist or physician in most studies $[8,9,11-13,15,17-19,21-25,27-29,30-34]$, to the comparison of pre- and postoperative hemoglobin concentrations in five studies $[14,16,20$, $26,30]$ and to the number of blood transfusions in three [8-10]. The volume of blood loss in particular papers is presented in Table III.

This heterogeneity markedly limited our ability to compare the results of various studies. However, some authors evaluated a significantly larger blood loss in TAH patients even with two methodological measures. Terai et al. [8] and Lu et al. [9] stated that patients subjected to TAH needed blood transfusion significantly more often than those who underwent TLH and also supported this thesis with the volume estimation of blood loss. Lu et al. [9] and Malzoni et al. [14] observed a greater decline in hemoglobin level postoperatively and greater volume loss.

In the Manchana et al. study [27] greater blood loss was determined in the TAH group ( $p<0.01$ ); however, an interesting correlation was discovered regarding patients' BMI. In a subset of women with $\mathrm{BMI}>30 \mathrm{~kg} / \mathrm{m}^{2}$, the degree of blood loss after TAH was greater than after TLH, whereas similar difference was not documented in normal-weight patients [27].

Not many papers have discussed the reason for greater blood loss during laparotomy. Yet according to O'Hanlan et al. [17], lesser blood loss during TLH was associated primarily with better visualization of the surgical field and hence with more favorable conditions to remove regional lymph nodes. However, they also pointed to larger size of the uterus in patients subjected to TAH as a potential contributor to greater intraoperative blood loss [17].

\section{Length of hospital stay}

Mean length of hospital stay after TLH was less than 4 days (range 1.2-14.7), as compared to more than 7 days (range 4-17.7) after TAH, with the differences being significant in all studies (Table III). In the series examined by O'Hanlan et al. [17], up to $25 \%$ of the patients were discharged home already within 24 hours after the procedure. According to the authors of publications included in this review, TLH is associated with shorter hospital stay due to shorter operative time, lesser blood loss and faster recovery. Boosz et al. [26] suggested that longer hospital stay after TAH observed in their series might be a consequence of the relatively high relaparotomy rate (11.9\%).

\section{Normalization of bowel function}

The recovery of bowel function has been addressed in only a single paper. Terai et al. [8] determined the mean time to the normalization of bowel function, i.e. to the first passage of flatus, at $1.6 \pm 0.6$ days for the TLH group and at $1.3 \pm 0.7$ days for the TAH group ( $p=0.11)$. Mean time to tolerance of a regular diet after TLH and TAH was $6.0 \pm 1.4$ and $6.6 \pm 3.0$ days, respectively $(p=0.27)$.

\section{Return to normal physical activity}

Only two papers focused on assessing the resumption of daily activities [12, 15]. According to Qvigstad and Lieng [12], patients after TLH and TAH returned to their normal physical activity after 2-3 and 5-6 weeks, respectively. However, those authors neither provided the definition of "normal physical activity" nor specified the method used to determine that parameter. In the study conducted by Mourits et al. [15], 6 weeks after the surgery, resumption of daily activities, determined with QoL scales - the Short Form-36 Health Survey (SF-36), the Body Image Scale (BIS), and the Visual Analogue Scale (VAS) - was observed in $76 \%$ of women after TLH and in $62 \%$ of patients after TAH. The same study did not demonstrate a statistically significant difference in the proportion of patients after TLH and TAH who resumed their professional activities at 6 weeks after surgery (22\% vs. $26 \%)$.

\section{Severity of postoperative pain}

The severity of postoperative pain was assessed in only four studies [8, 15, 27, 34]. Manchana et al. [27] and Bige et al. [34] estimated the severity of the pain with a visual analog scale (VAS). Manchana et al. [27] monitored the pain severity every 6 hours during the first 3 days after the surgery; their study showed that while patients subjected to TLH and TAH did not differ in terms of their pain estimates on postoperative day 1 , women who underwent the laparoscopic procedure reported less pain on subsequent days. In the study conducted by Bige et al. [34], patients subjected to TLH reported less pain throughout all days of postoperative hospital stay.

Terai et al. [8] and Mourits et al. [15] assessed the severity of pain based on the time of postoperative analgesia; this parameter was found to be shorter after TLH than after TAH (1.1 \pm 0.7 vs. 2.4 \pm 1.8 days). 


\section{Conversion to laparotomy during laparoscopy}

Conversion to laparotomy was necessary in only 139 out of 2,833 (4.91\%) women subjected to TLH. While no conversions were required in six studies $[8,10,14,20,21,33]$, the conversion rates reported by particular authors varied from 0 to $7 \%$. Based on the published data, the primary causes of conversion to TAH during TLH can be summarized as:

- intestinal injury $(4.6 \%)[30,34]$,

- too large uterus $(0.54 \%)[15,16,18,19]$,

- poor visualization of surgical field $(0.33 \%)[15,19]$,

- obesity $(0.24 \%)$ [15, 29-31],

- bleeding (0.22\%) [15, 22, 27, 34] (surgical injury to the inferior vena cava) [27],

- intraperitoneal adhesions (0.21\%) [18, 30, 31, 34],

- unspecified intraoperative complications (0.21\%) [16],

- peritoneal invasion (0.19\%) [17, 22, 31, 34],

- surgical injury to the urinary bladder (0.06\%) [27, 30],

- technical problems with laparoscopic equipment $(0.18 \%)[15,16]$,

- patient's intolerance to Trendelenburg position (0.06\%) [19],

- problems with anesthesia (0.03\%) [13] (hypercapnia).

Neither Santi et al. [22] nor Eisenkop et al. [31] identified patient's body mass index (BMI) as a factor enforcing the change of surgical approach. Moreover, Eisenkop et al. [31] demonstrated that conversion to laparotomy was not associated with patient's age or history of past surgery. According to Mourits et al. [15], some patients included in their series required conversion to laparotomy due to more than one cause.

The authors of five studies did not specify how many patients included in their series needed conversion to TAH during TLH $[9,24-26,28]$.

\section{Relaparotomy rates}

In most analyzed studies (15 studies, including $62.9 \%$ of the patients), the issue of relaparotomy was either dealt with inadequately or was not addressed at all; thus, the exact incidence of this complication is hard to estimate $[8,9,12,15,18$, $19,22-25,27-30,33]$. Moreover, none of the authors provided a definition of early and late relaparotomy. If mentioned at all, the relaparotomy was listed as one of postoperative complications, without detailed analysis of the problem. The spectrum of postoperative complications reported by some authors, i.e. dehiscence of surgical wound requiring repeat closure, or formation of abdominal hernia being eligible for surgical treatment, suggests that a certain proportion of their patients might require relaparotomy.
In studies that addressed the matter, relaparotomy was needed in $8.6 \%$ of patients subjected to $\operatorname{TLH}[9,11]$ and in $28.1 \%$ of women who underwent $\mathrm{TAH}$ [9]. Barwijuk and Jankowska reported only one case requiring reoperation, a patient with insulin-independent diabetes mellitus and $\mathrm{BMI}>30$ $\mathrm{kg} / \mathrm{m}^{2}$, in whom dehiscence of the surgical wound occurred at 7 days after TAH [20]. In the series analyzed by Boosz et al. [26], reoperation was necessary in 19 patients after TAH (11.9\%) and in one woman after TLH (0.9\%). The indications for relaparotomy included abdominal hernia $(n=5)$, evacuation of a lymphocele ( $n=5$, including one woman after TLH), infection of the surgical wound $(n=4)$, hemorrhage $(n=2)$, intestinal adhesions $(n=1)$, bleeding from a duodenal ulcer $(n=1)$ and hernia with the entrapment of the small bowel $(n=1)$ [26]. In the study conducted by Pellegrino et al. [13], reoperations were required due to formation of a hernia at the trocar site with resultant intestinal necrosis (one patient after TLH), dehiscence of the surgical wound ( 2 patients after TAH) and symptomatic hernia ( 2 patients after TAH). Insertion of drains in the trocar holes was shown to prevent formation of a lymphocele in patients subjected to TLH [20].

\section{Morbidity rates}

The most common complications after TAH (overall morbidity rate of $15 \%$ ) were inflammation of the surgical wound and fever (7.5\%) $[8,16,18$, 19, 21, 25, 27, 29, 30]. In turn, patients after TLH, with an overall morbidity rate of $8.1 \%$, most often suffered from abdominal hernias (4\%), surgical injuries to large vessels with resultant bleeding (3.9\%), and bowel obstruction (3.5\%) $[9,11,15,16$, $21,26,29-31]$. Since the incidence of postoperative abdominal hernia was analyzed in a small group of patients $(n=24)$, any conclusions about the risk of this complication should be formulated carefully. The list of less common complications (occurring in less than $1 \%$ of cases) of either TLH or TAH included Clostridium difficile infections, acute nephritis, herniation at the trocar site, inadvertent ligation of the ureters, and stroke [17-20, 23, 31].

According to O'Hanlan et al. [17], the vast majority of intraoperative complications related to TLH occurred during the initial two-thirds of the procedures performed by a given surgical team; consequently, intraoperative morbidity decreased with the increase in the slope of the learning curve. In turn, Obermiar et al. [16] demonstrated that intra- and postoperative complications were more frequent in patients subjected to regional and paraaortic lymphadenectomy.

\section{Long-term outcomes}

Overall survival and disease-free survival rates in patients operated on for endometrial malig- 
nancies were analyzed in only nine studies $[9,11$, 14, 21, 27, 28, 30, 33, 34] (Table IV). Comparative analysis of long-term outcomes in patients subjected to TLH and TAH did not show statistically significant differences in either overall or disease-free survival, although the assessment was limited to only six studies.

Five-year disease-free survival rates for patients subjected to TLH were between 85.3 and $97.1 \%$, as compared to between 88.5 and $96.3 \%$ in women who underwent TAH. Overall survival rates for patients operated on with these two techniques were $85.3-98.6 \%$ and $86.7-98.6 \%$, respectively $[9,11,14$, 21, 28, 30, 33, 34].

Recurrence rate was assessed in all mentioned papers but compared in six [9, 14, 21, 27, 33, 34]. The recurrence rates did not differ considerably across the surgical techniques and were in the range $4.2-11.1 \%$ for TLH and 5-11.9\% for TAH.

The most common sites of recurrence were vagina, peritoneum, liver and lungs; yet no study compared the site in relation to surgical techniques. None of the authors reported a recurrence at the trocar site.

Similarly, no statistically significant differences were observed in postoperative mortality rates, which were $4.3 \%$ for TLH and $5.4 \%$ for TAH [9, 11, $14,21,27,30,34]$. The most common causes of postoperative mortality were pulmonary embolism, sepsis during chemotherapy, bowel obstruction and radiation-induced bowel injury $[14,15,21,27,29,30$, 33, 34].

\section{Discussion}

Comparative analysis of TLH and TAH based on the results of 26 published studies was not infrequently challenging, due to their non-homogeneous research methodology. Another serious limitation of the studies, apart from four randomized studies and one matched study, was the lack of uniformity between the surgical groups in the patient- and tumor-related characteristics. Early or late outcomes of particular procedures cannot be well assessed if the surgery is more demanding due to more advanced stages of cancer, less favorable prognostic factors and more comorbidities of the patients. Another constraint is that a large proportion of studies did not even evaluate the homogeneity of the groups compared. These issues further hindered the interpretation of the results and made the analysis less objective.

All analyzed studies demonstrated that TLH is a safe and effective treatment option in endometrial cancer patients. This procedure is markedly less invasive than TAH. Moreover, TLH is associated with shorter hospital stay, faster recovery, lesser blood loss and fewer intra- and postoperative blood transfusions, reduced pain, and a lower reoperation rate than conventional TAH. However, considering several contraindications for laparoscopy, such as peritoneal invasion, cardiorespiratory failure, history of previous surgery and large size of the uterus, qualification for a given procedure needs to be preceded by a detailed evaluation.

Our analysis showed that TLH and TAH were associated with similar morbidity rates. Similarly, we did not find statistically significant procedurerelated differences in overall survival, disease-free survival or recurrence rates. Total abdominal hysterectomy was superior to TLH in terms of shorter operative time and steeper slope of the learning curve. According to some authors, however, the difference in the duration of both procedures may reflect longer time required for preparation of laparoscopic instruments.

Conversion to laparotomy was necessary in no more than one per twenty women subjected to $\mathrm{TLH}$, usually due to massive peritoneal invasion, obesity, hemorrhage or large size of the uterus. According to Walker et al., the frequency of conversions to laparotomy increased with patient's $\mathrm{BMI}$ and age [35].

Available evidence suggests that TLH is a safer treatment option than TAH in endometrial cancer. Women with endometrial malignancies frequently present with concomitant diseases, such as diabetes mellitus, cardiovascular diseases, etc. Considering all previously mentioned advantages of laparoscopy (faster recovery and earlier mobilization of patients, lesser demand for pain medications), this method seems to be a better treatment option in this group of patients. Moreover, it should be remembered that some severe comorbidities may constitute a contraindication for general anesthesia or surgery in the Trendelenburg position. Furthermore, some authors emphasized lower incidence of thromboembolism (pulmonary embolism and deep vein thrombosis) after TLH [17]. The surgeon performing the laparoscopic procedure uses a manipulator to better expose the uterine corpus in order to improve the accuracy of the resection and to prevent inadvertent ureteral injury. This raises concerns about the risk of potential spread of cancer cells to the vaginal cuff and peritoneal cavity via the fallopian tubes. However, available evidence suggests that uterine manipulation during TLH is not associated with increased likelihood of positive intraoperative peritoneal lavage cytology in endometrial cancer patients [32]. Moreover, many authors highlighted the role of TLH in endometrial cancer patients with concomitant obesity. Since the prevalence of overweight and obesity is still increasing, we may expect a growing incidence of endometrial cancer in this population. In one study, women subjected to $\mathrm{TAH}$ were older than those who gave their con- 
sent for TLH. This difference might be associated with the fact that younger patients generally prefer innovative methods of treatment which leaves a more esthetic scar [27]. Also another important aspect, improvement of general health status, needs to be emphasized; in one study, the proportion of patients who assessed their subjective health after TLH as better was higher than the analogous percentage of women who underwent TAH (44.4 vs. 29.0\%) [19]. Total laparoscopic hysterectomy is also associated with lesser risk of intraperitoneal adhesion formation, which may also have a considerable impact on the quality of life after the surgery. One study demonstrated that women subjected to TLH had better quality of life at 6 weeks after the procedure, which probably should be attributed to faster recovery, lesser pain and shorter hospitalization [35].

To summarize, available evidence shows clearly that TLH is superior to TAH in terms of the postoperative quality of life. However, wider acceptance of TLH as a treatment option in endometrial cancer still may be limited due to a relatively flat learning curve and longer operative times.

Nevertheless, according to some authors, the operators who gained experience in advanced laparoscopic techniques will likely benefit in future, as they will be better prepared for performing robotic surgery in patients with uterine malignancies.

\section{Conflict of interest}

The authors declare no conflict of interest.

\section{References}

1. Zwierko M. Epidemiology of endometrial malignancies. In: Bidziński M, Zwierko M, Markowska J, et al., editors. Endometrial cancers. Warsaw: Medical Center of Postgraduate Education in Warsaw; 2011. p. 5-25.

2. National Cancer Registry http://onkologia.org.pl/.

3. Gabryś M. Epidemiology and etiopathogenesis of endometrial cancer. In: Markowska J, editor. Oncological gynecology. Urban \& Partner Medical Publishing; 2006; p. 683-685.

4. Wojciechowska U, Didkowska J, Zatoński. Malignant neoplasms of the uterus (C54). In: Malignant neoplasms of the uterus. Warsaw: Oncology Center-Institute in Warsaw; 2008. p. 30-32.

5. Aporowicz M, Domosławski P, Czopnik P, Sutkowski K, Kaliszewski K. Perioperative complications of adrenalectomy - 12 years of experience from a single center/ teaching hospital and literature review. Arch Med Sci 2018; 14: 1010-9.

6. Markowska J, Wronkowski Z, Zwierko M. Epidemiology of reproductive organ cancers. In: Markowska J, editor. Oncological gynecology. Urban \& Partner Medical Publishing; 2002. p. 3-37.

7. Stroup D, Berlin J, Morton S, et al. Meta-analysis of observational Studies in Epidemiology: a proposal for reporting. Meta-analysis of Observational Studies in Epidemiology (MOOSE) group. JAMA 2000; 283: 2008-12.
8. Sokouti M, Sadeghi R, Pashazadeh S, et al. A systematic review and meta-analysis on the treatment of liver hydatid cyst using meta-MUMS tool: comparing PAIR and laparoscopic procedures. Arch Med Sci 2019; 15: 284-308.

9. Terai Y, Tanaka T, Sasaki H, et al. Total laparoscopic modified radical hysterectomy with lymphadenectomy for endometrial cancer compared with laparotomy. J Obstet Gynaecol Res 2014; 2: 570-5.

10. Lu Q, Liu H, Zhang Z, et al. Comparison of laparoscopy and laparotomy for management of endometrial carcinoma: a prospective randomized study with 11-year experience. J Cancer Res Clin Oncol 2013; 139: 1853-9.

11. Jung YW, Lee DW, Kim SW, et al. Robot-assisted staging using three robotic arms for endometrial cancer: comparison to laparoscopy and laparotomy at a single institution. J Surg Oncol 2010; 101: 116-21.

12. Corrado G, Cutillo G, Pomati G, et al. Surgical and oncological outcome of robotic surgery compared total laparoscopic and abdominal surgery in the management of endometrial cancer. Eur J Surg Oncol 2015; 141: 1074-81.

13. Qvigstad E, Lieng M. Surgical treatment of endometrial cancer and atypical hyperplasia: a trend shift from laparotomy to laparoscopy. Obstet Gynecol Int 2011; 2011: 829425.

14. Pellegrino A, Signorelli $M$, Fruscio $R$, et al. Feasibility and morbidity of total laparoscopic radical hysterectomy with or without pelvic lymphadenectomy in obese women with stage I endometrial cancer. Arch Gynecol Obstet 2009; 279: 655-60.

15. Malzoni M, Tinelli R, Cosentino F, et al. Total laparoscopic hysterectomy versus abdominal hysterectomy with lymphadenectomy for early-stage endometrial cancer: a prospective randomized study. Gynecol Oncol 2009; 112: 126-33.

16. Mourits MJE, Bijen CB, Arts HJ, et al. Safety of laparoscopy versus laparotomy in early-stage endometrial cancer: a randomised trial. Lancet Oncol 2010; 11: 763-71.

17. Obermair A, Janda M, Baker J, et al. Improved surgical safety after laparoscopic compared to open surgery for apparent early stage endometrial cancer: results from a randomised controlled trial. Eur J Cancer 2012; 48: 1147-53.

18. O'Hanlan K, Huang G, Garnier A, et al. Total laparoscopic hysterectomy versus total abdominal hysterectomy: cohort review of patients with uterine neoplasia. Journal of the Society of Laparoendoscopic Surgeons 2005; 9: 277-86.

19. Eisenhauer EL, Wypych KA, Mehrara BJ, et al. Comparing surgical outcomes in obese women undergoing laparotomy, laparoscopy, or laparotomy with panniculectomy for the staging of uterine malignancy. Ann Surg Oncol 2007; 14: 2384-91.

20. Boggess JF, Gehrig PA, Cantrell L, et al. A comparative study of 3 surgical methods for hysterectomy with staging for endometrial cancer: robotic assistance, laparoscopy, laparotomy. Am J Obstet Gynecol 2008; 199: 360.1-360.9.

21. Barwijuk A, Jankowska S. Is laparoscopic or abdominal hysterectomy with bilateral salpingo-oophorectomy more efficient in operative treatment of endometrial cancer? J Obstet Gynaecol 2005; 25: 703-5.

22. Gao H, Zhang Z. Laparoscopy versus laparotomy in the treatment of high-risk endometrial cancer. Medicine 2015; 94: 1245.

23. Santi A, Kuhn A, Gyr T, et al. Laparoscopy or laparotomy? A comparison of 240 patients with early-stage endometrial cancer. Surg Endosc 2010; 24:939-43. 
24. Lim PC, Kang E, Park DH. Learning curve and surgical outcome for robotic-assisted hysterectomy with lymphadenectomy: case-matched controlled comparison with laparoscopy and laparotomy for treatment of endometrial cancer. J Minim Invasive Gynecol 2010; 17: 739-48.

25. Berretta R, Gizzo S, Noventa M, et al. Quality of life in patients affected by endometrial cancer: comparison among laparotomy, laparoscopy and vaginal approach. Pathol Oncol Res 2015; 21: 811-6.

26. Bell MC, Torgerson J, Seshadri-Kreaden U, Suttle AW, Hunt S. Comparison of outcomes and cost for endometrial cancer staging via traditional laparotomy, standard laparoscopy and robotic techniques. Gynecol Oncol 2008; 111: 407-11.

27. Boosz A, Haeberle L, Renner SP, et al. Comparison of reoperation rates, perioperative outcomes in women with endometrial cancer when the standard of care shifts from open surgery to laparoscopy. Arch Gynecol Obstet 2014; 290: 1215-20.

28. Manchana T, Puangsricharoen P, Sirisabya N, et al. Comparison of perioperative and oncologic outcomes with laparotomy, and laparoscopic or robotic surgery for women with endometrial cancer. Asian Pac J Cancer Prev 2015; 16: 5483-8.

29. Chiou HY, Chiu LH, Chen CH, Yuan-Kuei Y, Chang CW, Liu WM. Comparing robotic surgery with laparoscopy and laparotomy for endometrial cancer management: a cohort study. Int J Surg 2015; 13: 17-22.

30. Barnett JC, Havrilesky LJ, Bondurant AE, et al. Adverse events associated with laparoscopy vs laparotomy in the treatment of endometrial cancer. Am J Obstet Gynecol 2011; 205: 143.1-143.6.

31. Coronado PJ, Herraiz MA, Magrina JF, Fasero M, Vidant JA Comparison of perioperative outcomes and cost of robotic-assisted laparoscopy, laparoscopy and laparotomy for endometrial cancer. Eur J Obstet Gynecol Reprod Biol 2012; 165: 289-94.

32. Eisenkop SM. Total laparoscopic hysterectomy with pelvic/aortic lymph node dissection for endometrial cancer - a consecutive series without case selection and comparison to laparotomy. Gynecol Oncol 2010; 117: 216-22.

33. Eltabbakh G, Mount S. Laparoscopic surgery does not increase the positive peritoneal cytology among women with endometrial carcinoma. Gynecol Oncol 2006; 100: 361-4.

34. Chu LH, Chang WCh, Sheu BCh. Comparison of the laparoscopic versus conventional open method for surgical staging of endometrial carcinoma. Taiwan J Obstet Gynecol 2016; 55: 188-92.

35. Bige O, Demir A, Saatli B, Koyuncuoglu M, Saygili U. Laparoscopy versus laparotomy for the management of endometrial carcinoma in morbidly obese patients: a prospective study. J Turk Ger Gynecol Assoc 2015; 16: 164-9.

36. Walker JL, Piedmonte MR, Spirtos NM. Laparoscopy compared with laparotomy for comprehensive surgical staging of uterine cancer: Gynecologic Oncology Group Study LAP2. J Clin Oncol 2009; 27: 5331-6. 